\title{
The role of convex probe endobronchial ultrasound-guided transbronchial needle aspiration in the diagnosis of hilar and mediastinal lesions
}

\author{
Laila A. Helalah ${ }^{a}$, Ashraf M. Madkour ${ }^{a}$, Nevine M.A. Elfattah ${ }^{a}$, \\ Rehab M. Mohammed ${ }^{\mathrm{a}}$, Ayman A.H. Farghaly ${ }^{\mathrm{c}}$, Ragaa A. Fawzy ${ }^{\mathrm{b}}$, \\ Asmaa M.A. Elasser ${ }^{d}$
}

\begin{abstract}
Background Endobronchial ultrasound-guided transbronchial needle aspiration (EBUS-TBNA) has been recently introduced as a new technique for sampling the hilar/ mediastinal lesions [lymph node (LN) enlargement or masses] with a potential to improve the diagnostic yield as it allows direct visualization of lesion beyond the tracheobronchial wall allowing real-time sampling. EBUSTBNA diagnostic yield has been satisfactory for both benign and malignant lesions.
\end{abstract}

Aim To evaluate the utility of convex probe EBUS-TBNA in the diagnosis of hilar and mediastinal lesions (LN enlargement or masses)

Patients and methods This is a prospective study in which EBUS-guided TBNA via a real-time ultrasound bronchoscope was used to diagnose 25 patients with mediastinal or hilar LN enlargement or masses.

Results EBUS-guided TBNA was performed on 15 patients with enlarged mediastinal/hilar LNs and 11 patients with mediastinal masses, achieving specific diagnosis in $73.3 \%$ $(11 / 15)$ and $81.8 \%(9 / 11)$, respectively. The overall diagnostic yield of EBUS-TBNA was $76 \%$ (19/25). Overall sensitivity was $82.6 \%$, specificity $100 \%$, positive predictive value $100 \%$, and

\section{Introduction}

Invasive sampling of mediastinal lesions is paramount for diagnosis and further treatment plans, and also the diagnosis of mediastinal lymphadenopathy is crucial in staging of lung cancer and/or extrathoracic malignancies [1]. Mediastinal lymphadenopathy constitutes a frequent diagnostic dilemma and may significantly alter treatment and prognosis, and sampling of intrathoracic lymphadenopathy has been most commonly and traditionally performed via mediastinoscopy, which is associated with a $1 \%$ complication rate and requirement for general anesthesia [2].

In recent years, alternative, less-invasive methods that are performed under local anesthesia with conscious sedation have gained popularity for collecting samples for cytological evaluation, such as endoscopic ultrasound-guided fine needle aspiration, but unfortunately, this technique does not allow access to the right paratracheal and hilar lymph nodes (LNs) [3]. Among these novel methods, endobronchial ultrasound (EBUS) stands out as a minimally invasive, safe method, negative predictive value $33.33 \%$. EBUS-TBNA procedure had no complications in $76 \%$ of cases.

Conclusion EBUS-TBNA is a minimally invasive, safe, yet still underutilized diagnostic technique with adequate diagnostic yield. Its nationwide application in the field of diagnostic bronchoscopy should be encouraged.

Egypt J Bronchol 2019 13:644-653

( 2020 Egyptian Journal of Bronchology

Egyptian Journal of Bronchology 2019 13:644-653

Keywords: endobronchial ultrasound-directed transbronchial needle aspiration, malignancy, mediastinal lesions, mediastinal lymph nodes, sarcoidosis, tuberculosis

Departments of, ${ }^{a}$ Chest Diseases, ${ }^{b}$ Pathology, Ain Shams University, ${ }^{\mathrm{C}}$ Department of Chest Diseases, Military Medical Academy. ${ }^{\mathrm{d}}$ Chest Department, Ministry of Health and Population, Chest Registrar, ElAbbassia Chest Hospital, Cairo, Egypt

Correspondence to Rehab M. Mohammed, MD, PhD, 54 Abdel Rahman Elrafaey Street, El Hegaz Square, Heliopolis, Cairo 11786, Egypt. Tel: +20 1223012 0418;

e-mail: dr.rehabmaher@gmail.com

Received: 27 June 2019 Accepted: 12 September 2019 Published: 21 January 2020

which can be performed in an outpatient setting, under sedation, being able to detect and collect material from $\mathrm{LNs}$ smaller than $1 \mathrm{~cm}$, and endobronchial ultrasound-guided transbronchial needle aspiration (EBUS-TBNA) allows access and sampling of paratracheal as well as subcarinal and hilar stations [4].

The technique has an established role in the diagnosis and staging of lung cancer, with a sensitivity of more than $90 \%$ [5]. Prospective data are now available on the utility of EBUS-TBNA in the diagnosis of sarcoidosis $[6,7]$, and a small series has also demonstrated a high diagnostic yield in patients with tuberculous lymphadenopathy [8].

Implementing this new technology for the first time, in one of Egyptian Ministry of Health and population

This is an open access journal, and articles are distributed under the terms of the Creative Commons Attribution-NonCommercial-ShareAlike 4.0 License, which allows others to remix, tweak, and build upon the work non-commercially, as long as appropriate credit is given and the new creations are licensed under the identical terms. 
tertiary care specialized chest hospitals, seems challenging in many aspects. The aim of this study was to present our initial experience to evaluate the role of EBUS-TBNA in the diagnosis of hilar and mediastinal lesions (LN enlargement or masses).

\section{Study design}

This is a prospective cross-sectional study, performed on 25 patients, at least 18 years old who presented with undiagnosed mediastinal or hilar LN enlargement or masses situated around the tracheobronchial airways as observed in their contrast-enhanced chest computed tomography $(\mathrm{CT})$ scans. The study was first conducted at El-Abbassia Chest Hospital during the period between March 2015 and July 2016, during which six cases were subjected to EBUS-TBNA, and then the study was stopped until July 2017 owing to irreparable technical problems in EBUS system (working channel) at El-Abbassia Chest Hospital, after which the study was then continued at Kobri El-Koba Military Hospital where EBUS-TBNA was performed on the remaining 19 cases for diagnosis of their hilar and/or mediastinal lesions.

Patients clinically unfit for bronchoscopy according to the international standards of practice [9] and patients who refused to perform the EBUS-TBNA procedure were excluded.

Informed consent was obtained from all patients or their relatives, and the study was approved by Institutional Ethical Committee Board of Faculty of Medicine, Ain Shams University.

\section{Before procedure}

All recruited patients were subjected to full demographic data and medical history taking, thorough clinical examination, contrast-enhanced CT scan of the chest (unfortunately, there was no standardization of radiology center; therefore, the measurement of the LNs/ mediastinal lesions size could not be performed in some cases owing to lack of measurement scales on CT cuts), coagulation profile, along with other laboratory investigation whenever needed, including kidney function, liver function, arterial blood gases, and complete blood count. Agents for anticoagulation, whenever indicated, were temporarily withheld before procedure to reduce bleeding risk [10].

At El-Abbassia Chest Hospital, the procedure was performed under general anesthesia for all six patients, whereas at Kobri El-Koba Military Hospital, premedication consisted of local administration of inhaled xylocaine $2 \%$ to the oropharynx and nostrils. Midazolam administration was left at the discretion of the bronchoscopist.

The procedure was performed in an equipped endoscopy suite according to the international standards of practice [11].

EBUS-guided TBNA was performed using a real-time ultrasound bronchoscope (EBUS Convex Probe BFUC180F; Olympus Medical Systems, Tokyo, Japan) and a dedicated ultrasound processor (Prosound $\alpha 7$ Premier; Hitachi-Aloka, Tokyo, Japan) at El-Abbassia Chest Hospital (Figs 1 and 2, respectively) versus Pentax EB-1970UK (Tokyo, Japan) and HI Vision Preirus (Hitachi-Aloka) at Kobri El-Koba Military Hospital.

Transbronchial needle biopsies were performed using single-use EBUS-TBNA ViziShot (Olympus Medical Systems) at El-Abbassia Chest Hospital versus a dedicated 22-G needle (EchoTip ProCore HD Ultrasound Biopsy Needle; Cook Medical, Limerick, Ireland) (Fig. 3), at Kobri El-Koba Military Hospital.

\section{During the procedure}

(1) Continuous monitoring of respiratory rate, oxygen saturation, cardiac rhythm, rate, blood pressure, and any complication occurring during procedure was recorded.

(2) Rapid endobronchial assessment was done either by conventional fiberoptic bronchoscopy (FOB) or by the EBUS-FOB to search for endobronchial lesion and for further bronchial lavage and/or endobronchial biopsies if needed.

(3) Assessment of all EBUS-accessible LNs and mediastinal lesions was done according to $\mathrm{LN}$ mapping and bronchoscopic landmarks of representative LN stations [12]; vascular structures were avoided using the Doppler function applied immediately before needle puncture. Under direct ultrasound guidance, the EBUS-TBNA from the lesion was done using 22$\mathrm{G}$ needle (LN and lesions diameter could not be measured in all cases).

(4) Steps of EBUS-TBNA were done according to Mehta et al. [13]. A syringe was attached to the proximal end to perform aspiration through the needle. Samples were collected from the LN in the shape of cells or tissue fragments by back and forth motion as the aspiration continued using jabbing method. There was no pathologist present during the procedure for rapid on-site cytological 


\section{Figure 1}

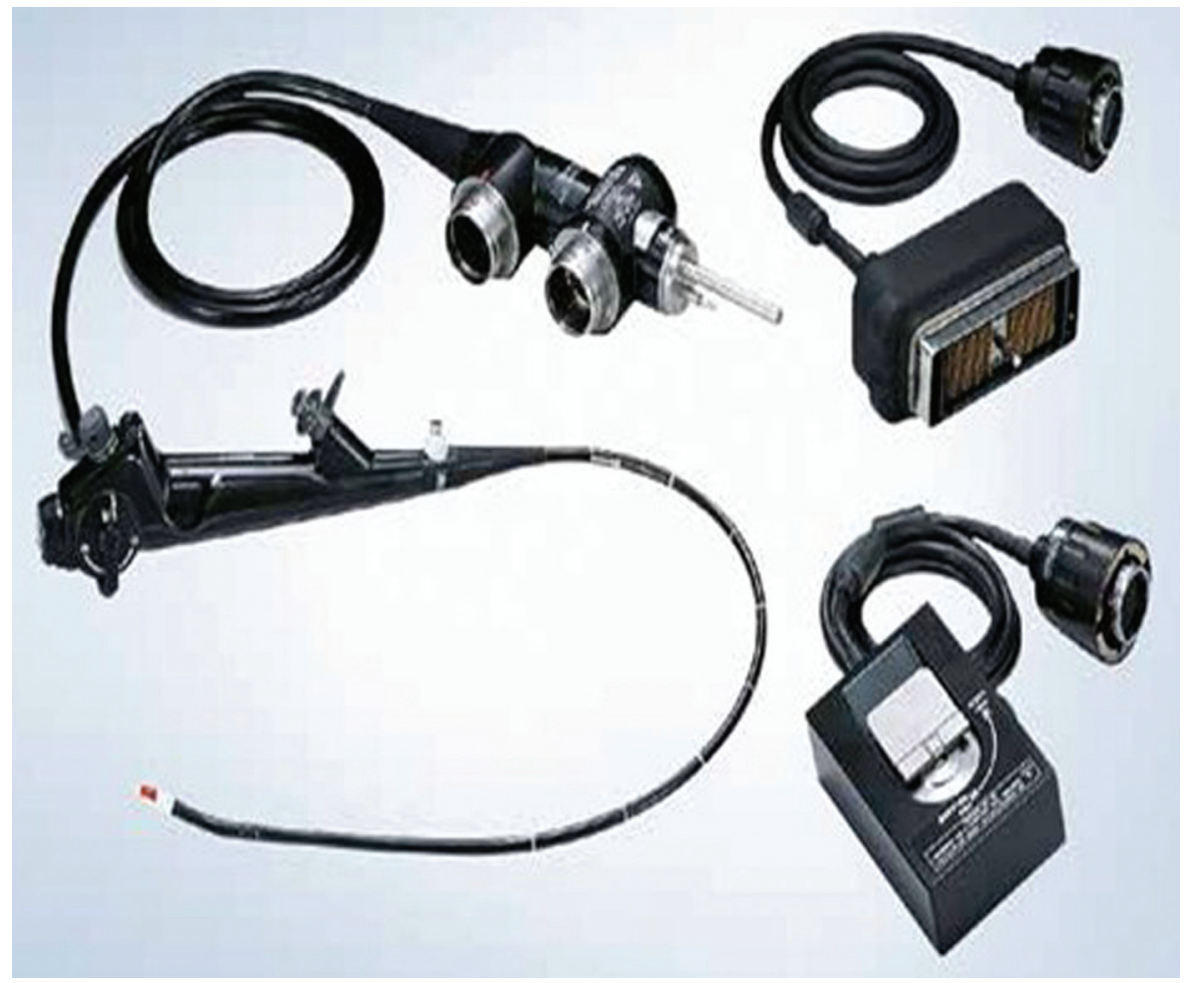

Endobronchial ultrasound convex probe BF-UC180F.

\section{Figure 2}

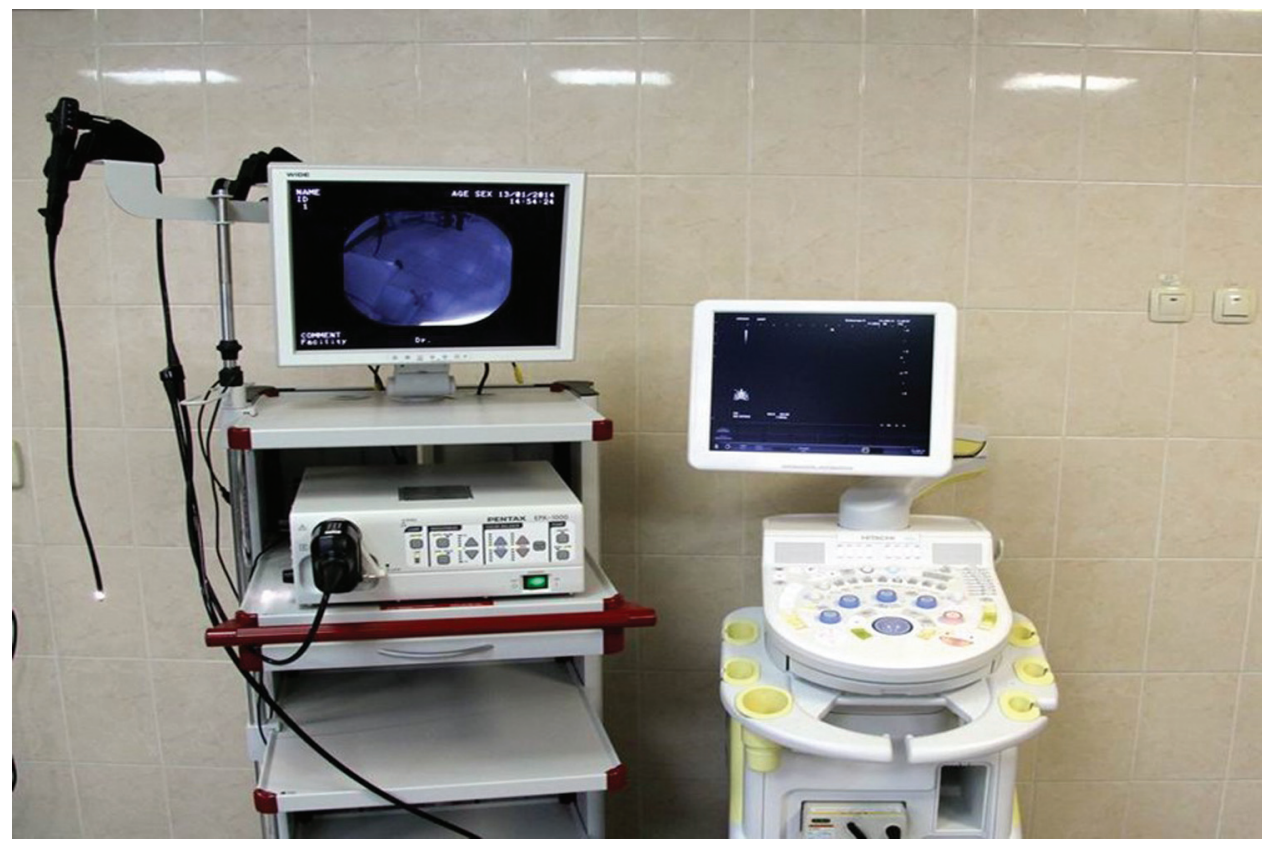

Endobronchial ultrasound convex probe BF-UC180F with ultrasound processor prosound $\alpha 7$ Premier.

examination (ROSE). Depending on the amount of collected material, the bronchoscopist assessed the adequacy for each sample, and if it was necessary, the EBUS-TBNA procedure was repeated from the same area until enough material was collected. Number of passes by the needle per lesion was recorded. The aspirate from the needle was then smeared onto glass slides by the experienced operator, air dried, and fixed immediately with $95 \%$ alcohol, and if there were histological core biopsies, they were fixed with $10 \%$ neutral buffered formalin. 
Figure 3

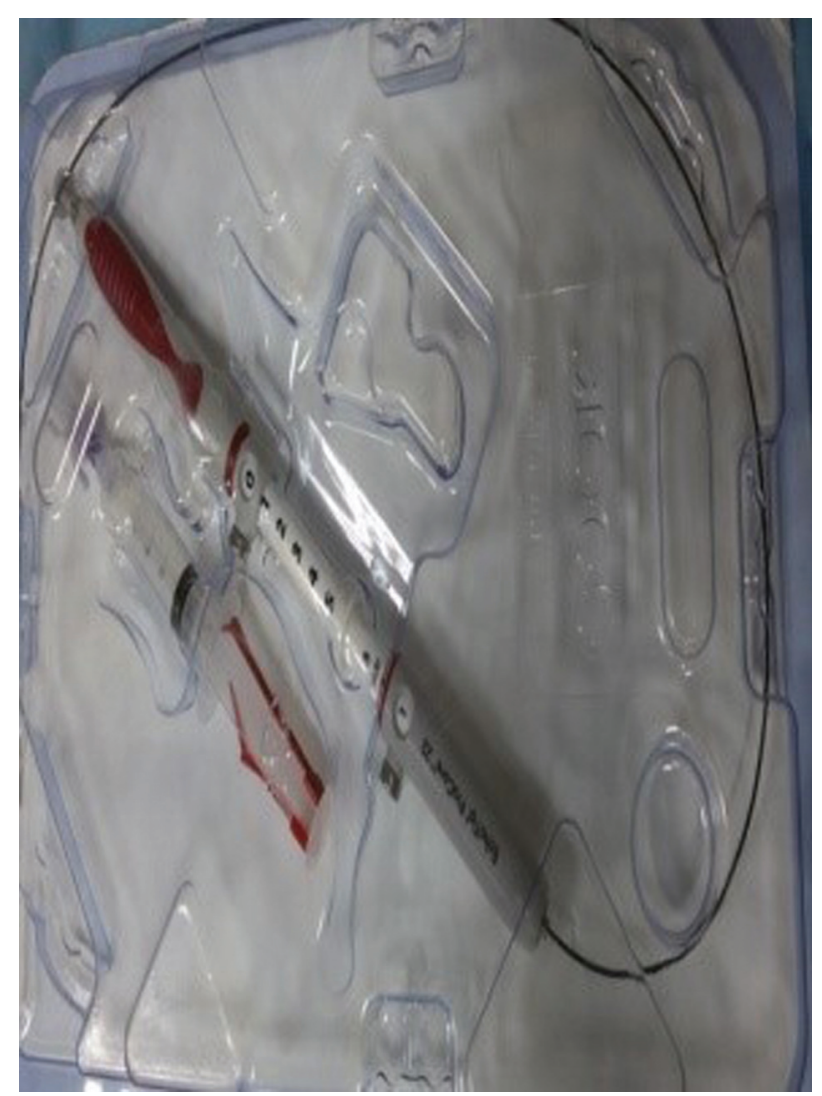

EchoTip ProCore HD ultrasound biopsy needle, $22 \mathrm{G}$.

(5) Images were taken for documentation of the procedure.

(6) The procedure time from the start of EBUS insertion was also documented, and it depended on the number of needle passes, time needed for preparation of the slides, and patient co-operation, especially if only local anesthesia was applied.

(7) Endobronchial lesions seen via FOB were biopsied and sent for histopathology as well.

(8) None of the patients developed any procedurerelated serious complications.

\section{After the procedure}

(1) Postprocedure chest radiograph was routinely performed to exclude any procedure-related complications.

(2) Reaching a specific diagnosis was considered as gold standard; therefore, when cytological results were positive of specific diagnosis [malignancy or other diagnoses as tuberculosis (TB), sarcoidosis], this was accepted as evidence, and the patients were treated accordingly.

(3) When histopathological results did not provide specific diagnosis, patients were either referred for mediastinoscopy or put under clinical follow- up for 6 months according to the clinical judgment.

\section{Statistical analyses}

The collected data were coded, tabulated, and statistically analyzed using statistical package for the social sciences (SPSS) software version 18.0 (IBM Corp., Chicago, Illinois, USA). Descriptive statistics were done for quantitative data as minimum and maximum of the range as well as mean $\pm \mathrm{SD}$, whereas qualitative data were analyzed as number and percentage. In qualitative data, inferential analyses were done using $\kappa$ test for agreement between paired categorical data. The level of significance was taken at $P$ value less than 0.05 .

Diagnostic characteristics such as sensitivity, specificity, positive predictive value (PPV), negative predictive value (NPV), and diagnostic accuracy (DA) were calculated using standard formulae on a perpatient basis when a specific final diagnosis was reached.

\section{Results}

A total of 25 patients requiring diagnostic bronchoscopy either for mediastinal/hilar LN enlargement or mediastinal mass as presented in their contrastenhanced chest $\mathrm{CT}$ scans were recruited in this study. Baseline features of the enrolled patients are summarized in Table 1: the mean $\pm \mathrm{SD}$ age was $57.2 \pm 11.9$ years, and $60 \%(15 / 25)$ were males. Ninety-six $(24 / 25)$ of the enrolled patients complained of dyspnea, whereas cough and hemoptysis were the least presenting complaints (one case for each, $4 \%$ ).

Mediastinal/hilar LN enlargement was the most frequent encountered CT scan finding [15/25 (60\%) patients], whereas mediastinal masses were found in $11 / 25$ (44\%) cases, and coexisting parenchymal lesions were seen in $4 / 25(16 \%)$.

EBUS-TBNA-related events are summarized in Table 2. The most frequent LN station punctured by EBUS-TBNA was subcarinal (21 times) versus left main bronchus (25 times) in cases of mediastinal lesions. The number of passes by EBUS-TBNA needle ranged from 2 to 7 per lesion per procedure, with a median of 4, and time elapsed from the start of EBUS insertion to the end of the procedure ranged from 30 to $45 \mathrm{~min}$. No recorded complications were seen in 19/25 (76\%) cases, and minimal bleeding occurred in 6/25 (24\%) patients, which was self-limiting and needed no interference. 
Table 1 General characteristics of the studied cases

\begin{tabular}{lc}
\hline Variables & \\
\hline Age (years) & $33-80$ \\
$\quad$ Range & $57.2 \pm 11.9$ \\
Mean \pm SD & $15 / 10(60 /$ \\
Sex (male/female) $[N(\%)]$ & $40)$ \\
& \\
Main complaint $[N(\%)]$ & $24(96)$ \\
Dyspnea & $6(24)$ \\
Chest pain & $5(20)$ \\
Hoarseness & $5(20)$ \\
Fever & $3(12)$ \\
Weight loss & $1(4)$ \\
Cough & $1(4)$ \\
Hemoptysis & \\
CT scan findings $[N$ (\%)] & $11(44)$ \\
Bilateral LN enlargement (mediastinal/hilar) & $9(36)$ \\
Mediastinal mass & $3(12)$ \\
Bilateral LN enlargement (mediastinal/hilar) and & $1(4)$ \\
coexisting parenchymal lesion & \\
Bilateral LN enlargement (mediastinal/hilar) and & \\
mediastinal mass & \\
lesion & \\
\hline
\end{tabular}

CT, computed tomography; LN, lymph node.

The primary outcome was obtaining a specific (definitive) diagnosis on a per-patient basis. Definitive diagnosis could be obtained in 23 (92\%) patients: $12 / 25$ (48\%) were malignant, whereas $11 / 25$ (44\%) cases had benign lesions, where 9/25 (36\%) were diagnosed with sarcoidosis and two patients had TB. The remaining two (8\%) patients had nonconclusive results; one (4\%) patient had EBUS-TBNA cytology result suspicious of malignancy (atypical cells) but he refused any further evaluation via surgery or mediastinoscopy, and the second (4\%) patient had chronic nonspecific inflammation by both the EBUS-TBNA cytology and histopathology of endobronchial biopsies but unfortunately he died shortly after without reaching a specific diagnosis (Table 3).

Tables 4 and 5 summarize the method of final diagnosis of the studied cases where EBUS? TBNA was overall diagnostic in 19 of 25 studied cases, with $76 \%$ diagnostic rate (yield): 10/12 (83.33\%) malignant cases, 7/9 (77.77\%) sarcoid cases, and two (100\%) tuberculous cases. Moreover, EBUS-guided TBNA achieved a specific (definitive) diagnosis in $73.3 \%$ $(11 / 15)$ patients who presented with enlarged mediastinal/hilar LNs and 81.8\% (9/11) patients who presented with mediastinal masses.

On the contrary, endobronchial biopsies via FOB were diagnostic in 6/12 (50\%) malignant cases and 4/9 (44.44\%) sarcoid cases, and mediastinoscopy was the diagnostic tool in 1/9 (11.11\%) sarcoid cases.
Table 2 Endobronchial ultrasound-transbronchial needle aspiration-related events

\begin{tabular}{lc}
\hline LN stations punctured via EBUS-TBNA & $N$ \\
\hline LNs & 21 \\
Subcarinal (7) & 10 \\
Left lower paratracheal LNs (4) & 10 \\
Left hilar LNs (10) & 5 \\
Right hilar LNs (10) & 3 \\
Right lower paratracheal LNs (4) & \\
Mediastinal lesion & 25 \\
Left main bronchus & 6 \\
Right main bronchus & 5 \\
Left lower paratracheal region & 85 \\
Total & $2-7$ \\
Number of needle passes by EBUS-TBNA per lesion & 4 \\
Range & \\
Median & $19(76)$ \\
Anesthesia [N (\%)] & 6 (24) \\
Local & \\
General & \\
\hline Lymph node stations are named according to international \\
guidelines [12]. EBUS-TBNA, endobronchial ultrasound-guided \\
transbronchial needle aspiration; LNs, lymph node station. \\
\hline
\end{tabular}

Table 3 Final histopathological diagnosis of the studied cases

\begin{tabular}{lc}
\hline Diagnosis & $N(\%)$ \\
\hline Malignancy & $12(48)$ \\
Squamous cell carcinoma & $6(24)$ \\
Adenocarcinoma & $1(4)$ \\
Un-differentiated & $1(4)$ \\
Small cell carcinoma & $3(12)$ \\
Lymphoma & $1(4)$ \\
Benign & $11(44)$ \\
Sarcoidosis & $9(36)$ \\
Tuberculosis & $2(8)$ \\
Nonconclusive & $2(8)$ \\
Atypical cells (suspicious of malignancy) & $1(4)$ \\
Chronic nonspecific inflammation & $1(4)$ \\
\hline
\end{tabular}

The overall sensitivity, specificity, PPV, and NPV of EBUS-TBNA were 82.6, 100, 100, and 33.33\%, respectively, with an overall DA of $84 \%$ (Table 6).

Finally, on further analysis of the diagnostic validity (performance) of EBUS-TBNA in different diseases, the technique showed sensitivity of $83.3 \%$, specificity of $100 \%$, PPV of $100 \%$, and NPV of $86.7 \%$ in diagnosis of malignancy. However, the EBUSTBNA had sensitivity, specificity, PPV, and NPV of $77.7,100,100$, and $88.8 \%$, respectively, in diagnosing sarcoidosis. EBUS-TBNA had sensitivity, specificity, PPV, and NPV of $100 \%$ for all in TB diagnosis (Table 7).

Table 8 shows that there was almost perfect agreement between EBUS-TBNA diagnosis and final diagnosis 
Table 4 Methods of final diagnosis of the studied cases

\begin{tabular}{lccc}
\hline & $\begin{array}{c}\text { Malignancy }(n=12) \\
{[N(\%)]}\end{array}$ & $\begin{array}{c}\text { Sarcoidosis }(n=9) \\
{[N(\%)]}\end{array}$ & $\begin{array}{c}\text { TB }(n=2) \\
{[N(\%)]}\end{array}$ \\
\hline EBUS-TBNA only & $6(50)$ & $4(44.44)$ & $2(100)$ \\
Both EBUS-TBNA and FOB & $4(33.33)$ & $3(33.33)$ & $12(48)$ \\
FOB only & $2(16.7)$ & $1(11.11)$ & $7(28)$ \\
Mediastinoscopy & & $1(11.11)$ & $3(12)$ \\
Undiagnosed & & & $2(100)$ \\
\hline
\end{tabular}

EBUS-TBNA, endobronchial ultrasound guided transbronchial needle aspiration; FOB, fiberoptic bronchoscopy; TB, tuberculosis.

Table 5 Overall detection rate (diagnostic yield) of endobronchial ultrasound-transbronchial needle aspiration in diagnosis

\begin{tabular}{|c|c|c|c|}
\hline \multirow[t]{2}{*}{ Diagnosis by EBUS-TBNA } & \multicolumn{2}{|c|}{ Final diagnosis } & \multirow[t]{2}{*}{ Total $[N(\%)]$} \\
\hline & Diagnosed & Not diagnosed & \\
\hline Diagnosed & 19TP & OFP & $19(76)$ \\
\hline Not diagnosed & $4 \mathrm{FN}$ & $2 T N$ & $6(24)$ \\
\hline Total & $23(96)$ & $2(4)$ & $25(100)$ \\
\hline
\end{tabular}

EBUS-TBNA, endobronchial ultrasound guided transbronchial needle aspiration; FN, false negative; FP, false positive; TN, true negative; $\mathrm{TP}$, true positive.

Table 6 Diagnostic validity of endobronchial ultrasoundguided transbronchial needle aspiration

\begin{tabular}{lcc}
\hline Parameters & Value (\%) & $95 \% \mathrm{Cl}$ \\
\hline Sensitivity & 82.6 & $61.22-95.05$ \\
Specificity & 100 & $15.81-100$ \\
DA & 84 & $63.92-95.46$ \\
PPV & 100 & - \\
NPV & 33.33 & $17.02-54.92$ \\
\hline
\end{tabular}

$\mathrm{Cl}$, confidence interval; DA, diagnostic accuracy; EBUS-TBNA, endobronchial ultrasound-guided transbronchial needle aspiration; NPV, negative predictive value; PPV, positive predictive value.

regarding malignancy, sarcoidosis, and $\mathrm{TB}$, as $\kappa$ score was $0.84,0.91$, and 1 , respectively.

\section{Representative cases}

Figures 4 and 5 .

\section{Discussion}

The introduction of EBUS-TBNA into the world of diagnostic bronchoscopy has caused a major shift in the sampling of mediastinal lymphadenopathy. Over the past decade, researchers have studied the diagnostic performance of EBUS-TBNA. In this prospective cross-sectional study, we aimed to evaluate the role of EBUS-TBNA in the diagnosis of hilar and mediastinal lesions (LN enlargement or masses) located around the tracheobronchial airways as shown in contrast-enhanced chest CT scans.

In this study, the overall diagnostic yield (detection rate) of EBUS-TBNA was 76\%. The technique achieved a specific (definitive) diagnosis in $73.3 \%$ $(11 / 15)$ of patients having enlarged mediastinal/hilar LN and $81.8 \%(9 / 11)$ of patients with mediastinal masses. These findings are comparable to a great extent with those of Safwat et al. [14], the earliest Egyptian experience with EBUS-TBNA (conducted in 2005-2006,using radial probe), although they reported higher diagnostic yield (83\%), and specific diagnosis was reached in $82.3 \%(14 / 17)$ of examined LN and $84.6 \%(11 / 13)$ of examined mediastinal masses. Moreover, a second Egyptian another study in 2018 [15] reported an even higher diagnostic yield (89.2\%).

Our results show an overall sensitivity of $82.6 \%$, specificity of $100 \%$, PPV of $100 \%$, NPV of $33.33 \%$ and an overall DA of $84 \%$. On comparing these results with those of Vaidya et al. [16] who reported similar EBUS-TBNA sensitivity, specificity, and PPV for EBUS-TBNA (89 and 100, 100\%, respectively, whereas NPV in their study was higher than in ours (53\%). Ye et al. [17] found similar specificity and PPV (100\% for each), but higher sensitivity, NPV, and overall accuracy of $(95,93$, and 97\%, respectively); these higher values reported in their study may be explained by the larger study population size compared with the small sample size of this study and the that they calculated the diagnostic performance of the EBUS-TBNA procedure on a per-node basis in addition to sampling from 225 LNs, which was 2.5 times higher than that of our study (85 nodes).

Regarding the diagnostic validity of EBUS-TBNA in the diagnosis of malignancy, sensitivity was $83.3 \%$, specificity 100\%, PPV 100\%, and NPV 86.7\%, with a DA of $92 \%$. These findings are comparable to those of Navani et al. [18] who performed EBUS-TBNA on 
Table 7 Diagnostic validity of endobronchial ultrasound-guided transbronchial needle aspiration in diagnosis of malignancy, sarcoidosis, and tuberculosis

\begin{tabular}{|c|c|c|c|c|c|c|}
\hline & \multicolumn{2}{|c|}{ Malignancy } & \multicolumn{2}{|c|}{ Sarcoidosis value } & \multicolumn{2}{|c|}{ TB value } \\
\hline & Value (\%) & $95 \% \mathrm{Cl}$ & Value (\%) & $95 \% \mathrm{Cl}$ & Value (\%) & $95 \% \mathrm{Cl}$ \\
\hline Sensitivity & 83.3 & $51.6-97.9$ & 77.7 & $47.3-99.7$ & 100 & $15.8-100$ \\
\hline Specificity & 100 & 75.3-100 & 100 & $80.5-100$ & 100 & $85.2-100$ \\
\hline DA & 92 & $74-99$ & 92 & $79.6-99.9$ & 100 & $86.3-100$ \\
\hline PPV & 100 & $69.2-100$ & 100 & $59.0-100$ & 100 & $15.8-100$ \\
\hline NPV & 86.7 & $59.5-98.3$ & 88.8 & $72.7-99.9$ & 100 & $85.2-100$ \\
\hline
\end{tabular}

$\mathrm{Cl}$, confidence interval; EBUS-TBNA, endobronchial ultrasound-guided transbronchial needle aspiration; FN, false negative; FP, false positive; TB, tuberculosis; TN, true negative; TP, true positive.

Table 8 Comparison of endobronchial ultrasound-guided transbronchial needle aspiration results with final diagnosis

\begin{tabular}{|c|c|c|c|c|c|c|}
\hline \multirow[t]{2}{*}{ Final diagnosis } & \multicolumn{4}{|c|}{ Diagnosis via EBUS-TBNA } & \multirow[t]{2}{*}{$\kappa(95 \% \mathrm{Cl})$} & \multirow[t]{2}{*}{$P$ value } \\
\hline & TP & FP & TN & FN & & \\
\hline Malignant & $10(40)$ & 0 & $13(52)$ & $2(8)$ & $0.84(0.63-1.00)$ & $<0.001^{*}$ \\
\hline Sarcoidosis & $7(28)$ & 0 & $16(64)$ & $2(8)$ & $0.91(0.72-1.00)$ & $<0.001^{*}$ \\
\hline TB & $2(8)$ & 0 & $23(92)$ & 0 & $1.00(1.00-1.00)$ & $<0.001^{*}$ \\
\hline
\end{tabular}

Percentages are from the total number of cases (25 cases). $\mathrm{Cl}$, confidence interval; EBUS, endobronchial ultrasound; FN, false negative; FP, false positive; TB, tuberculosis; TBNA, transbronchial needle aspiration; TN, true negative; TP, true positive. *Highly significant $P$ value.

160 patients with intrathoracic lymphadenopathy coexisting with extrathoracic malignancy, reporting a sensitivity, NPV for malignancy, and overall accuracy of 87,73 , and $88 \%$, respectively. Kennedy et al. [19] found sensitivity, specificity, PPV, and NPV for EBUS-TBNA of 91, 100, 100, and 92.9\%, respectively, in their study on 211 patients with suspected lymphoma.

Higher diagnostic sensitivity of EBUS-TBNA in detecting malignancy was reported by Nakajima et al. [20] and Verma et al. [21] (94 and 91\%, respectively).

On the contrary, on evaluating the diagnostic performance of EBUS-TBNA in diagnosing benign lesions, our study results showed that in diagnosis of sarcoidosis, EBUS-TBNA had a diagnostic yield of 77.77\% (7/9 cases). A recent Egyptian study [22] investigating the value of EBUS-TBNA in diagnosis of sarcoidosis reported a lower diagnostic yield (62.9\%). This study resulted in a sensitivity of $77.7 \%$, specificity of $100 \%$, PPV of $100 \%$, NPV of $88.8 \%$, and accuracy of $92 \%$. The reported sensitivity in our study was slightly higher than that reported by Tournoy et al. [23] and lower than that found by Verma et al. [21] (71 and 86.5\%, respectively), a finding which may be attributed to the difference in disease prevalence.

Regarding the diagnosis of TB, EBUS-directed TBNA showed a sensitivity of $100 \%$, specificity of
$100 \%$, PPV of $100 \%$, and NPV of $100 \%$. Yet, these results are biased as there were only two TB cases enrolled in the study, which definitely does not reflect the actual prevalence of TB in our country.

Despite the small number of cases with sarcoidosis and TB in the present work ( 9 and 2 cases, respectively), the high diagnostic yield of EBUS-TBNA in diagnosis of these two diseases favors its use as a first diagnostic tool in suspected cases, and this matches the current international guidelines, which strongly recommend EBUS-TBNA as a first choice in diagnosing suspected cases of sarcoidosis and TB [24].

In this study, EBUS-TBNA procedure was fairly tolerated by the recruited participants with no encountered complications in $76 \%$ of cases, and only minimal bleeding occurred in $24 \%$, which was selflimiting requiring no interference.

There are some points of weakness in our study which may have influenced the diagnostic yield and the diagnostic validity. These points lie mainly in the small sample size of the study population and second point is the inability to properly assess the exact size of the punctured LNs as many of the radiology centers only report the presence of enlarged lymphadenopathy without mentioning the exact dimensions, and during the procedure, the exact cross diameters of $\mathrm{LNs}$ were difficult to measure mostly because the outer borders of the LNs were sometimes beyond the EBUS range and 

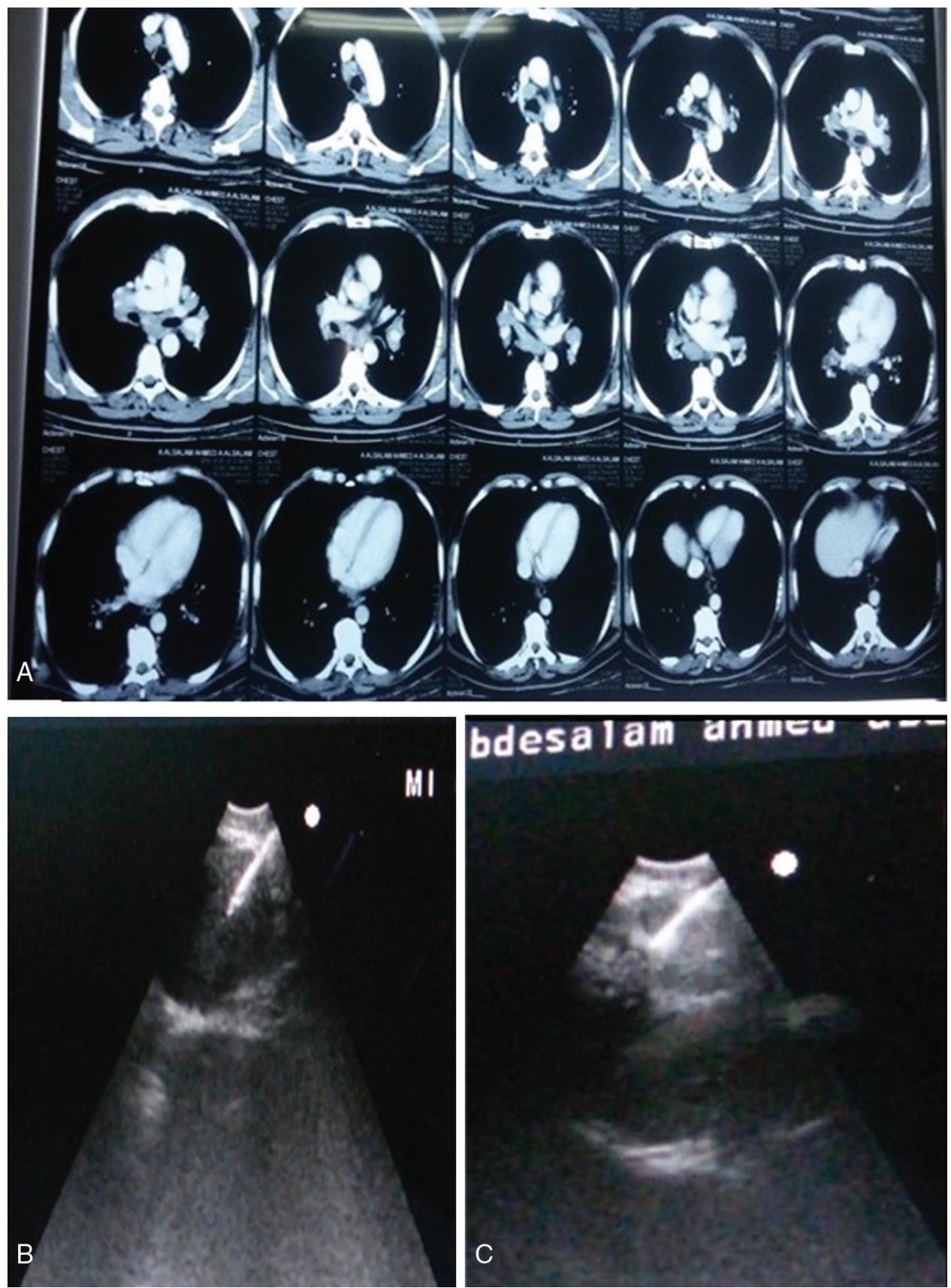

Chest computed tomography scan revealed multiple enlarged mediastinal lymphadenopathy involving right paratracheal, perivascular, aortopulmonary, and bilateral hilar. Endobronchial ultrasound-TBNA taken from left and right subcarinal lymph node (LN) (7) and left hilar LNs (10). Cytology results are consistent with sarcoidosis.

partly because of the difficulty encountered in the measuring technique, as the majority of cases (19/ $25)$ were performed under local anesthesia, with or without conscious sedation with a spontaneously breathing patient with respiratory movements, coughing, and clearing of secretions, which caused difficulty in acquisition of optimal LN dimensions. Moreover, the fact that we were compelled to change the location of the study and use different EBUS system as well as different operators, all of which may have influenced the results reached.
Another point to mention was the unavailability of ROSE during the procedure, and although several EBUS-TBNA researches in literature were conducted without ROSE, yet ROSE has add-on advantages in many aspects, mainly in ensuring adequacy and sufficiency of samples, increasing DA of EBUSTBNA [25]. We overcame this point by following the recent guidelines, which suggest that in the absence of ROSE, a minimum of three separate needle passes be performed [23] (number of needle passes per lesion in our study ranged from 2 to 7 , with a median of 4). 


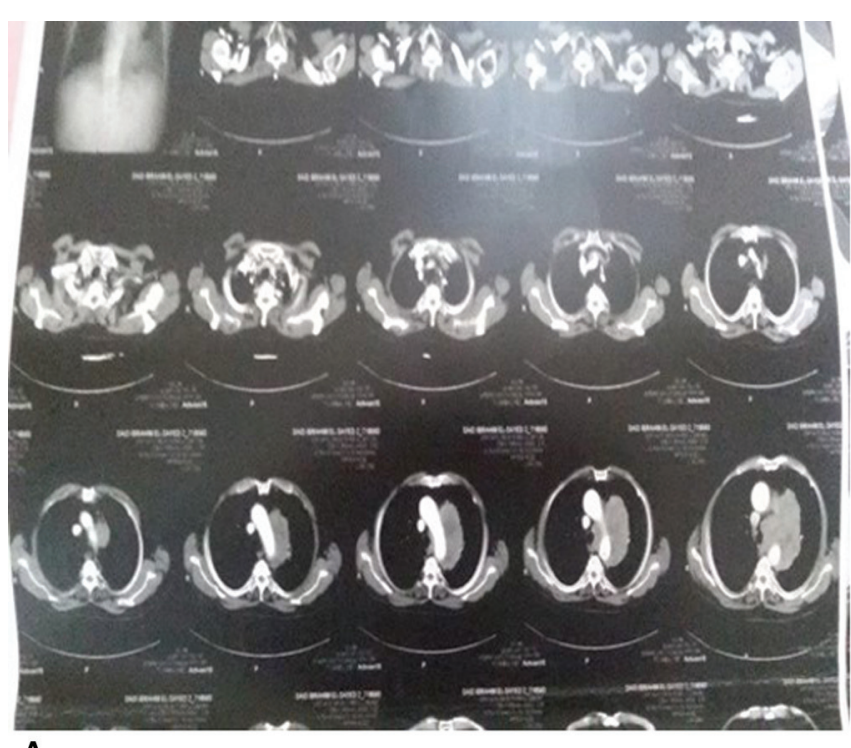

A

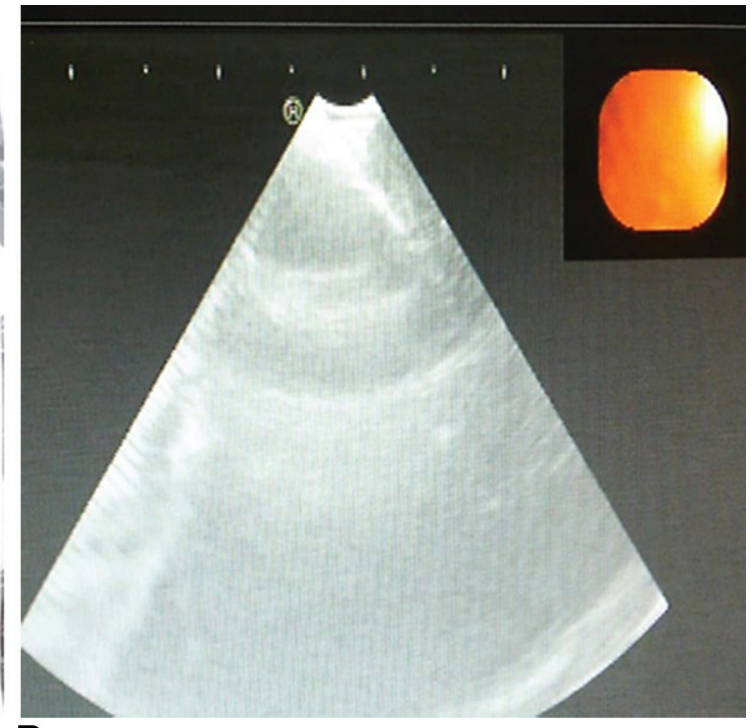

B

Chest computed tomography scan showing left mediastinal central mass $12 \times 8 \times 10 \mathrm{~cm}$ adherent to aortic arch and left pulmonary artery. Endobronchial ultrasound-transbronchial needle aspiration taken from subcarinal (7), left lower paratracheal lymph nodes (L4).

Moreover, 21/45 (43\%) of accessed LNs were subcarinal which are fairly easy to access and are known to have high diagnostic yield [14]. This may have influenced the results as well.

Limitations to our study include the high cost of the EBUS system, rendering it difficult to purchase by many centers in our country, not to mention the relatively frequent technical errors, which are rather difficult to repair with high maintenance expenses, which forced us to change our location from ElAbbassia Chest Hospital to Kobri El-Koba Military Hospital owing to the irreparable damage that occurred in the working channel of the EBUS bronchoscope in the former hospital.

Finally, the results of this study add to the accumulating data in literature highlighting the importance of incorporating EBUS-TBNA in routine assessment of enlarged mediastinal/hilar lymphadenopathy as well as in mediastinal masses. It permits more and smaller nodes to be sampled in an even safer and under vision manner than conventional TBNA and provides an alternative to the classical gold standard procedure (mediastinoscopy). In addition, EBUS-TBNA can access hilar nodal stations that could be difficult to be accessed by mediastinoscopy, compelling the operator to shift even to thoracotomy. Moreover, EBUS-TBNA permits safer approach to LNs situated beside blood vessels. Furthermore, it can be done under local anesthesia and can be easily repeated without the need of postprocedure hospitalization. All of which are considered very appealing advantages.

\section{Conclusion}

EBUS-TBNA is a promising method for sampling mediastinal/hilar lesions (LNs or masses), with significant diagnostic yield and minimal complications.

\section{Financial support and sponsorship}

Nil.

\section{Conflicts of interest}

There are no conflicts of interest.

\section{References}

1 Pillot G, Siegel BA, Govindan R. Prognostic value of fluorodeoxyglucose positron emission tomography in non-small-cell lung cancer: a review. $J$ Thorac Oncol 2006; 1:152-159.

2 Lemaire A, Nikolic I, Petersen T. Nine-year single center experience with cervical mediastinoscopy: complications and false negative rate. Ann Thorac Surg 2006; 82:1185-1189.

3 Navani N, Spiro SG, Janes SM. Mediastinal staging of NSCLC with endoscopic and endobronchial ultrasound. Nat Rev Clin Oncol 2009; 6:278-286.

4 Whitson BA, Groth SS, Maddaus MA. Surgical assessment and intraoperative management of mediastinal lymph nodes in non-smallcell lung cancer. Ann Thorac Surg 2007; 84:1059-1065.

5 Navani N, Nankivell M, Nadarajan P. The learning curve for EBUS-TBNA. Thorax 2011; 66:352-353.

6 Tremblay A, Stather DR, Maceachern P, Khalil M, Field SK. A randomized controlled trial of standard vs endobronchial ultrasonography-guided transbronchial needle aspiration in patients with suspected sarcoidosis. Chest 2009; 136:340-346.

7 Navani N, Booth HL, Kocjan G, Falzon M, Capitanio A, Brown JM, et al. Combination of endobronchial ultrasound-guided transbronchial needle aspiration with standard bron-choscopic techniques for the diagnosis of 
stage | and stage || pulmonary sarcoidosis. Respirology 2011; 16:467-472.

8 Hassan T, McLaughlin AM, O'Connell F, Gibbons N, Nicholson S, Keane J. EBUS-TBNA performs well in the diagnosis of isolated thoracic tuberculous lymphadenopathy. Am J Respir Crit Care Med 2011 183:136-137.

9 Jalil B, Yasufuku K, Maqbul Khan A. Uses, limitations, and complications of endobronchial ultrasound. Proc (Bayl Univ Med Cent) 2015; 28:325-330.

10 Ernst A, Eberhardt R, Wahidi M, Becker HD, Herth FJ. Effect of routine clopidogrel use on bleeding complications after transbronchial biopsy in humans. Chest 2006; 129:734-737.

11 Du Rand IA, Barber PV, Goldring J, Lewis RA, Mandal S, Munavvar M, et al. British Thoracic Society guidelines for advanced diagnostic and therapeutic flexible bronchoscopy in adults. Thorax 2011; 66:iii1-iii21.

12 Yasufuku K. EBUS-TBNA bronchoscopy. In: Ernst A, Herth JF, editors. Endobronchial ultrasound. An Atlas and practical guide. First edition. New York, NY: Springer Science and ?Business Media LLC. 2009. pp. 119-141.

13 Mehta AC, Wang KP, Turner JF. Teaching conventional transbronchia needle aspiration a continuum. Ann Am Thorac Soc 2013; 10:685-689.

14 Safwat T, Khattab A, EL Haddad S, Mostafa Y, Korraa E, Madkour A, et al. Endobronchial ultrasound-directed transbronchial needle aspiration in diagnosis of mediastinal lesions: initial Egyptian experience. $J$ Bronchology Interv Pulmonol 2009; 16:18-21.

15 Ahmed MAI. Evaluation of endobronchial ultrasound-guided transbronchial needle aspirate (EBUS-TBNA) in mediastinal lesions sampling. Mansoura: Doctor Degree Thesis, Mansoura University, Mansoura, Egypt 2018.

16 Vaidya PJ, Saha A, Kate AH, Pandey K, Chavhan VB, Leuppi JD. Diagnostic value of core biopsy histology and cytology sampling of mediastinal lymph nodes using 21-gauge EBUS-TBNA needle. $J$ Cancer Res Ther 2016; 12:1172-1177.
$17 \mathrm{Ye} \mathrm{T}, \mathrm{Hu} \mathrm{H}$, Luo X, Chen $\mathrm{H}$. The role of endobronchial ultrasound guided transbronchial needle aspiration (EBUS-TBNA) for qualitative diagnosis of mediastinal and hilar lymphadenopathy: a prospective analysis. BMC Cancer 2011; 11:100.

18 Navani N, Nankivell M, Woolhouse L, Harrison R, Munavvar M, Oltmanns $U$, et al. Endobronchial ultrasound-guided transbronchial needle aspiration for the diagnosis of intrathoracic lymphadenopathy in patients with extra thoracic malignancy. J Thorac Oncol 2011; 6:1505-1509.

19 Kennedy MA, Jimenez CA, Bruzzi JF, Mhatre AD, Lei X, Giles FJ, et al. Endobronchial ultrasound-guided transbronchial needle aspiration in the diagnosis of lymphoma. Thorax 2008; 63:360-365.

20 Nakajima T, Yasufuku K, Fujiwara T, Chiyo M, Sekine Y, Shibuya K, et al. Endobronchial ultrasound-guided transbronchial needle aspiration for the diagnosis of intrapulmonary lesions. J Thorac Oncol 2008; 3:985-988.

21 Verma A, Jeon K, Koh W, Suh G, Chung M, Kim H, et al. Endobronchial ultrasound-guided transbronchial needle aspiration for the diagnosis of central lung parenchymal lesions. Yonsei Med J. 2013; 54:672-678.

22 ABU Baker MA. Endobronchial ultrasound in diagnosis of undiagnosed pulmonary sarcoidosis. Tanta: Master's Degree Thesis, Tanta University, Tanta, Egypt; 2018.

23 Tournoy K, Bolly A, Aerts J, Pierard P, Pauw R, Leduc D, et al. The value of endoscopic ultrasound after bronchoscopy to diagnose thoracic sarcoidosis. Eur Respir J 2010; 35:1329-1335.

24 Wahidi MM, Herth F, Yasufuku K, Shepherd RW, Yarmus L, Chawla M, et al. Technical aspects of endobronchial ultrasound-guided transbronchial needle aspiration. Chest 2016; 149:816-835.

25 Bediwy AS, Zamzam K, Hantira M, El-Sharawy D, EISaqa A, Zamzam Y The value of rapid on-site evaluation during endobronchial ultrasoundguided transbronchial needle aspiration in the diagnosis of mediastinal lesions. Egypt J Bronchol 2017; 11:336-341. 